\title{
EFFECT OF GOOD CORPORATE GOVERNANCE AND SUSTAINABILITY REPORT DISCLOSURE STRUCTURE ON COMPANY VALUE AND ECONOMIC ADDED VALUE AS INTERVENING VARIABLE
}

\author{
Erika Astriani Aprilia \\ Univesity of Pamulang \\ Ariyanto.erika@gmail.com
}

\begin{abstract}
The purpose of this study to test the effect of Good Corporate Governance Structure, Sustainability Reporting Disclosure, and Firm Value on the Economic Value Added as an intervening variable. This study uses secondary data from the period 2010-2015 to firms listed at Indonesia Exchange. The sampling technique used purposive sampling. Tools to process the data using SPSS 23.The results showed Board of Commissioners Size, Independent Commissioner, Institutional Ownership have a significant impact on Firm Value while Board of Directors Size, Audit Committee, Managerial Ownership does not have effect on Firm Value. Board of Directors Size and Board of Commissioners Size have an effect on Economic Value Added and Firm Value, but Economic Value Added not an intervening variable between Board of Directors Size and Board of Commissioners Size. , Independent Commissioner has not effect on Economic Value Added, but Economic value added is an intervening variable between Independent Commissioner and Firm Value. Audit Committee does not effect on Economic Value Added and Firm Value, but Economic value added is an intervening variable between Audit Committee and Firm Value. Managerial ownership does not have effect on Economic Value Added and Firm Value, but Economic value added is an intervening variable between Managerial ownership and Firm Value. Institusional Ownership does not have effect on Economic Value Added and Economic Value Added is not intervening variable between Institusional Ownership and Firm Value. Sustainability Report Disclosure has an effect on Economic Value Added and Firm Value.
\end{abstract}

Keyword : Good Corporate Governance Structure, Sustainability Report Disclosure, Economic Value Added, Firm Value

\section{INTRODUCTION}

The value of the company going public is reflected in the price of its shares. The size of the stock price is basically determined by the strength between demand and supply, which is influenced by many factors, both the company's internal factors that issue shares and the company's external factors (Sudana, 2011: 88). One of the increases in company value is influenced by financial performance, especially on profitability (Rahayu, 2010). 
Good or effective company performance will be able to show the amount of added value that will be generated so that it is reflected in the increase in company value measured by Economic Value Added (EVA). In addition to financial performance factors that are oriented to shareholder value, maximization of company value can be achieved if the company pays attention to stakeholder stakeholders. Managers realize that public awareness has shifted, business is expected to not only prioritize single bottom line, namely profit, but begin to consider its impact on the environment (planet) and improve people's welfare (people). The suitability of business activities with the values that exist in the community will help companies to achieve long-term goals, namely sustainable benefits. The implementation of good corporate governance is considered to maximize the overall management functions of the company and lead to trust from investors that the company has been managed effectively and professionally.

Ghozali and Chariri (2014) state that a company is not an entity that only operates for its own sake but must provide benefits to its stakeholders (shareholders, creditors, consumers, suppliers, government, society, analysts and other parties). Thus, the existence of a company is strongly influenced by the support provided by stakeholders to the company. So that the company has a dependency on stakeholders to be able to carry out its functions and activities.

\section{LITERATURE REVIEW}

\subsection{The effect of Sustainability Report Disclosure has an effect on Economic Value Added and Company Value \\ With the sustainability report} disclosure conducted by the company, it is hoped that it can provide clear evidence that the production process carried out by the company is not only profit oriented but also takes into account the surrounding environment so as to increase stakeholder trust through increased investment that has an impact on increasing corporate profits. this condition indicates that disclosure of sustainability report can increase public confidence which results in increased company profits. The Arjowo (2013) and Safitri (2015) research shows that sustainability report disclosure influences performance. Ghozali and Chariri (2007) explain that companies are bound by social contracts with the community, where survival and growth are based on the final output (output) that can be given to the community. Acceptance from the community (legitimacy) is expected to increase the value of the company through a good corporate image which in turn will affect sales and increase in company profits (Soelistyoningrum and Prastiwi, 2011). Disclosure of the implementation of social responsibility to the workforce is also responded positively by stakeholders who not only can increase the company's average stock price, but also can improve employee welfare and loyalty and reduce employee turnover so that it can lead to increased company productivity (Ernst \& Young, 2013). Therefore, continuous disclosure of reports in important social dimensions and affect the financial performance of the organization. This was confirmed by the results of the study of Burhan and Rahmanti (2012) which showed that disclosure of social dimension sustainability had a significant impact on financial performance.

\subsection{Effect of Economic Value Added on Company Values}

The company's performance as measured by the economic value added shows the results of a more comprehensive calculation by involving 
more factors to assess the company's performance. Management is required to be able to manage the company's wealth as optimally as possible in creating added value for the company not only in the short term but in the long term where the company is run with the assumption that it will continue.

Good corporate performance will be attractive to investors and promises to creditors that the company is able to survive and generate profits in the long run.

In the research of Nugraha and Bachtiar, it shows the opposite, where the economic value added does not show any influence on the value of the company. It is inversely proportional to the research conducted by Rofiandri (2012) and Septiyani (2014) who found that the economic value added had an effect on the value of the company.

\section{RESEARCH METHOD}

\subsection{Population}

The population in this study were companies listed on the Indonesia Stock Exchange (IDX), which numbered 355 companies.

The research sample is a company that during the period 20102015 publishes financial statements that meet the following criteria:

Tabel 1: Procedure for Determining Criteria in Sample Selection

\begin{tabular}{|c|l|c|}
\hline No & \multicolumn{1}{|c|}{ Criteria } & Number \\
\hline 1 & $\begin{array}{l}\text { Companies listed on the Indonesia Stock Exchange in } \\
2010-2015\end{array}$ & 355 \\
\hline 2 & $\begin{array}{l}\text { Companies that do not publish continuous reports during } \\
2010-2015 \text { in a row }\end{array}$ & $(336)$ \\
\hline 3 & $\begin{array}{l}\text { The company publishes an annual report (annual report } \\
\text { stated in foreign currencies) }\end{array}$ & 16 \\
\hline Number of companies sampled & 6 \\
\hline Number of study periods & $\mathbf{9 6}$ \\
\hline
\end{tabular}

Source: Indonesia Stock Exchange. Data is processed by researcher, 2017

\subsection{Analytical Technique}

The analytical method to find out the independent variables that have a significant effect on company value on companies listed on the Indonesia Stock Exchange in 2010-2015, namely Board of Directors Size, Board of Commissioners Size, Independent Commissioner, Audit Committee, Managerial Ownership, Institutional Ownership, Sustainability Report Disclosure is used the general equation of multiple linear regression for seven independent variables using path analysis with 2 SLS, i.e. (Gujarati, 1993).

$Z: \alpha 0+\beta 1 X 1+\beta 2 X 2+\beta 3 X 3+\beta 4$ $\mathrm{X} 4+\beta 5 \times 5+\beta 6 \mathrm{X6}+\beta 7 \mathrm{X} 7++\varepsilon 1$
$Y: \alpha 0+\beta 1 X 1+\beta 2 X 2+\beta 3 X 3+\beta 4$

$X 4+\beta 5 X 5+\beta 6 X 6+\beta 7 X 7+\beta 8 Z+\varepsilon 1$

\subsection{Hypothesis testing}

3.3.1 coefficient of determination test $\left(\mathbf{R}^{2}\right)$

The coefficient of determination $\left(\mathrm{R}^{2}\right)$ is used to measure how far the model's ability to explain the variation of the dependent variable. According to Ghozali (2011), the coefficient of determination is between 0 and 1 . A small $\mathrm{R}^{2}$ value means that the ability of independent variables to explain variations in the dependent variable is very limited. A value close to one means that the independent variables provide almost all the information needed to predict the dependent variable. 


\subsection{2 t-test}

According to Ghozali (2011) The t test statistic shows how far the influence of one independent variable individually in explaining variations in the independent variable. Comparing the $p$ value with a significance level of 0.05 , it can be determined whether $\mathrm{HO}$ is rejected or accepted ( $\mathrm{H} 0$ is accepted if $\mathrm{p}$ value $>0.05, \mathrm{H} 0$ is rejected if $\mathrm{p}$ value $<0.05)$.

\subsubsection{F test}

The F statistical test is performed to show that the independent variables

included in the model have a joint effect on the dependent variable.

\subsubsection{Path Analysis}

Testing of hypotheses can be done by path analysis. Path analysis (path analysis) is the use of regression analysis to estimate causality between predetermined variables (Ghozali, 2005).

\section{RESULTS AND DISCUSSION}

\subsection{Deskriptive Analysis}

Table 2: Descriptive Statistics

\begin{tabular}{|l|r|r|r|r|r|}
\hline & $\mathrm{N}$ & Minimum & Maximum & \multicolumn{1}{c|}{ Mean } & Std. Deviation \\
\hline UDD & 96 & 4,0 & 11,0 & 7,000 & 1,6796 \\
UDK & 96 & 5,0 & 11,0 & 6,573 & 1,3038 \\
KI & 96 & 1,0 & 5,0 & 2,917 &, 8905 \\
KA & 96 & 2,0 & 7,0 & 3,990 & 1,0906 \\
KPMJ & 96 &, 00000 &, 00411 &, 0002979 &, 00075039 \\
KINS & 96 &, 165 &, 994 &, 54271 &, 300054 \\
SRDI & 96 &, 133 &, 978 &, 53044 &, 247515 \\
EVA & 96 & $-382120,1$ & 3467046,5 & 504680,339 & 773697,7855 \\
TOBIN'S Q & 96 &, 2974 & 6,3239 & 1,726702 &, 9755588 \\
Valid N (listwise) & 96 & & & & \\
\hline
\end{tabular}

Source: Processed, 2017

4.2 Classic assumption test 4.2.1 Normality Test

Table 3. One-Sample Kolmogorov-Smirnov Test

\begin{tabular}{|c|c|c|}
\hline & & Unstandardized Residual \\
\hline $\begin{array}{l}\text { N } \\
\text { Normal Parameters } \\
\text { Most Extreme Differences } \\
\text { Test Statistic } \\
\text { Asymp. Sig. (2-tailed) }\end{array}$ & $\begin{array}{l}\text { Mean } \\
\text { Std. Deviation } \\
\text { Absolute } \\
\text { Positive } \\
\text { Negative }\end{array}$ & $\begin{array}{r}96 \\
, 0000000 \\
, 26724796 \\
, 066 \\
, 066 \\
-, 061 \\
, 066 \\
, 200^{\mathrm{c}, \mathrm{d}}\end{array}$ \\
\hline $\begin{array}{l}\text { a. Test distribution is Norn } \\
\text { b. Calculated from data. } \\
\text { c. Lilliefors Significance } \\
\text { d. This is a lower bound o }\end{array}$ & rue significa & \\
\hline
\end{tabular}




\subsubsection{Heteroscedacity test}

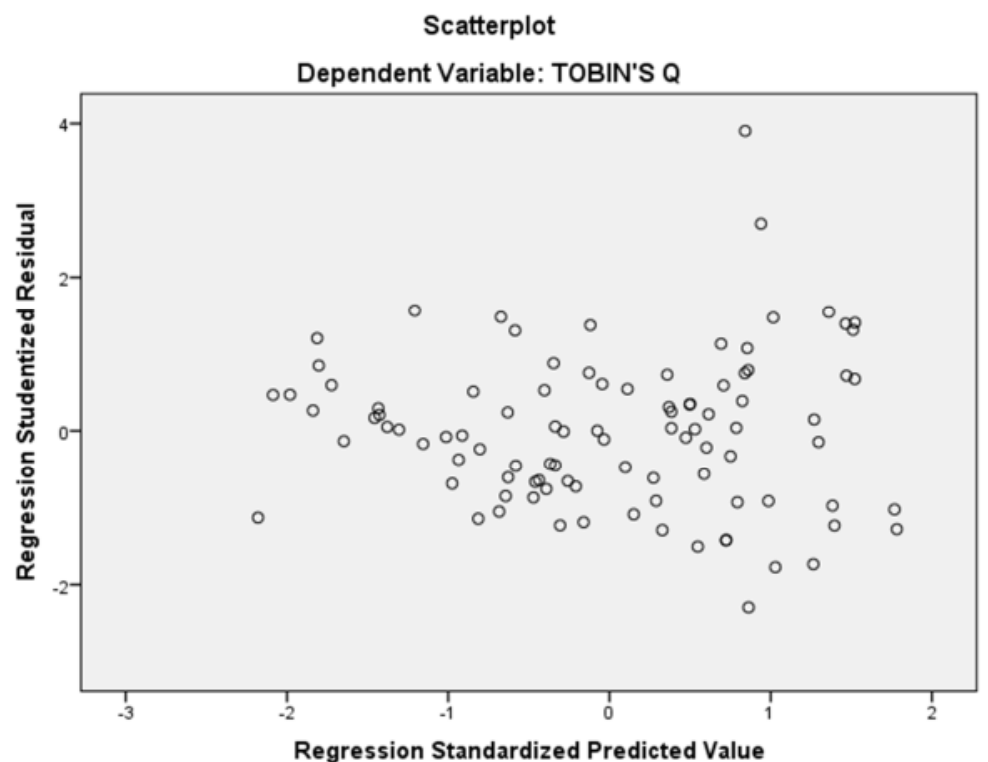

1.2.3 Autocorrelation Test

Table 4. Autocorrelation Test

\begin{tabular}{|l|r|r|r|r|r|}
\hline Model & $\mathrm{R}$ & R Square & $\begin{array}{c}\text { Adjusted R } \\
\text { Square }\end{array}$ & $\begin{array}{c}\text { Std. Error of the } \\
\text { Estimate }\end{array}$ & Durbin-Watson \\
\hline 1 &, $669^{\mathrm{a}}$ &, 448 &, 404 &, 293469 & 1,474 \\
\hline
\end{tabular}

a. Predictors: (Constant), SRDI, KA, KPMJ, UDK, UDD, KINS, KI

b. Dependent Variable: EVA

4.2.4. Multicoleniarity Test

Table 5. Multicoleniarity Test

\begin{tabular}{|c|c|c|c|c|c|c|c|}
\hline \multirow[b]{2}{*}{ Model } & \multicolumn{2}{|c|}{$\begin{array}{l}\text { Unstandardized } \\
\text { Coefficients }\end{array}$} & \multirow{2}{*}{$\begin{array}{c}\text { Standardized } \\
\text { Coefficients } \\
\text { Beta }\end{array}$} & \multirow[b]{2}{*}{$\mathrm{t}$} & \multirow[b]{2}{*}{ Sig. } & \multicolumn{2}{|c|}{$\begin{array}{c}\text { Collinearity } \\
\text { Statistics }\end{array}$} \\
\hline & $\mathrm{B}$ & Std. Error & & & & Tolerance & VIF \\
\hline 1 (Constant) & $-34,225$ & 41,525 & &,- 824 &, 412 & & \\
\hline UDD &, 685 &, 130 & ,555 & 5,276 & ,000 & 566, & 1,765 \\
\hline UDK &,- 618 & , 182 &,- 383 & $-3,391$ & ,001 & ,492 & 2,030 \\
\hline KI & ,262 & , 169 & , 179 & 1,550 & , 125 & ,473 & 2,115 \\
\hline KA & , 185 & , 119 & , 128 & 1,549 & , 125 & ,925 & 1,081 \\
\hline KPMJ & 56,875 & 58,655 & ,079 & ,970 & ,335 & ,939 & 1,065 \\
\hline KINS &, 117 & , 188 & ,063 & ,623 & ,535 & ,619 & 1,615 \\
\hline SRDI &,- 566 & 187 &,- 253 & $-3,025$ & ,003 & ,895 & 1,118 \\
\hline
\end{tabular}

a. Dependent Variable: EVA

4.3 Hypothesis Testing

4.3.1 Regression Analysis 
Model analysis is used to find out how much donations are given by independent variables, namely, Board of Directors Size, Board of Commissioners Size, Independent Commissioner, Audit Committee, Managerial Ownership, Institutional Ownership, Sustainability Report Disclosure, and Economic Value Added. In the regression analysis the model 1 structural equation is as follows:

$$
Z=\alpha^{0}+\beta_{1} X_{1}+\beta_{2} X_{2}+\beta_{3} X_{3}+\beta_{4} X_{4}+\beta_{5} X_{5}+\beta_{6} X_{6}+\beta_{7} X_{7}++\varepsilon_{1}
$$

\subsubsection{F-test}

Table 6 F EVA

ANOVA $^{\mathrm{a}}$

\begin{tabular}{|c|c|c|c|c|c|c|}
\hline Model & Sum of Squares & Df & Mean Square & $\mathrm{F}$ & Sig. & Kesimpulan \\
\hline 1 Regression & 6,140 & 7 &, 877 & 10,184 &, $000^{\mathrm{b}}$ & $\mathrm{H}_{1}$ diterima \\
\hline Residual & 7,579 & 88 & ,086 & & & \\
\hline Total & 13,719 & 95 & & & & \\
\hline
\end{tabular}

a. Dependent Variable: EVA

b. Predictors: (Constant), SRDI, KA, KPMJ, UDK, UDD, KINS, KI

Table 7 Determination coefficient test EVA

\begin{tabular}{|ll|r|}
\hline Equation 1 & Multiple R &, 669 \\
& R Square &, 448 \\
& Adjusted R Square &, 404 \\
& Std. Error of the Estimate &, 293 \\
\hline
\end{tabular}

\subsubsection{T-test}

Table 8 t-test EVA

\begin{tabular}{|c|c|c|c|c|c|c|c|}
\hline & & \multicolumn{2}{|c|}{$\begin{array}{c}\text { Unstandardized } \\
\text { Coefficients }\end{array}$} & \multirow[b]{2}{*}{ Beta } & \multirow[b]{2}{*}{$\mathrm{t}$} & \multirow[b]{2}{*}{ Sig. } & \multirow[b]{2}{*}{ Conclusion } \\
\hline & & $\mathrm{B}$ & Std. Error & & & & \\
\hline \multirow[t]{8}{*}{$\begin{array}{l}\text { Equatio } \\
\text { n } 1\end{array}$} & $\begin{array}{l}\text { (Consta } \\
\text { nt) }\end{array}$ & $-34,225$ & 41,525 & &,- 824 & ,412 & \\
\hline & UDD & ,685 &, 130 &, 555 & 5,276 & ,000 & $\mathrm{H}_{2}$ accepted \\
\hline & UDK &,- 618 &, 182 &,- 383 & $-3,391$ & ,001 & $\mathrm{H}_{3}$ rejected \\
\hline & $\mathrm{KI}$ & ,262 & , 169 & , 179 & 1,550 & , 125 & $\mathrm{H}_{4}$ rejected \\
\hline & KA &, 185 &, 119 & , 128 & 1,549 & , 125 & $\mathrm{H}_{5}$ rejected \\
\hline & KPMJ & 56,875 & 58,655 & ,079 & ,970 & ,335 & $\mathrm{H}_{6}$ rejected \\
\hline & KINS &, 117 & , 188 & ,063 & ,623 & ,535 & $\mathrm{H}_{7}$ rejected \\
\hline & SRDI &,- 566 & , 187 &,- 253 & $-3,025$ & ,003 & $\mathrm{H}_{8}$ accepted \\
\hline
\end{tabular}

The results of data processing in model 1 can be concluded with the following equation:

$Z=\alpha^{0}+\beta_{1} X_{1}+\beta_{2} X_{2}+\beta_{3} X_{3}+\beta_{4} X_{4}+\beta_{5} X_{5}+\beta_{6} X_{6}+\beta_{7} X_{7}++\varepsilon_{1}$ 
EVA $=0,555$ UDD $-0,383$ UDK + 0,179 KI + 0,128 KA + 0,079 KPMJ + 0,063 KINST $-0,253$ SRDI $+\varepsilon_{1}$

4.4. Effect of Board of Directors Size, Board of Commissioners Size, Independent Commissioner, Audit Committee, Managerial Ownership, Institutional Ownership, Sustainability Report Disclosure of Corporate Value through or not through the variable Economic Value Added

Tabel 9: Intervening and non Intervening variables

\begin{tabular}{|l|l|c|c|c|}
\hline No. & \multicolumn{1}{|c|}{ Effect } & $\begin{array}{c}\text { Direct } \\
\text { Effect }\end{array}$ & Indirect Effects & Decision \\
\hline 1 & $\begin{array}{l}\text { Size of the Board of Directors -> } \\
\text { Corporate Value through } \\
\text { Economic c Value Added as an } \\
\text { Intervening variable }\end{array}$ & 0,032 & $-0,032$ & $\begin{array}{c}\text { Not } \\
\text { intervening }\end{array}$ \\
\hline 2 & $\begin{array}{l}\text { Size of the Board of } \\
\text { Commissioners -> Corporate } \\
\text { Value through Economic Value } \\
\text { Added as an Intervening variable }\end{array}$ & 0,284 & 0,0927 & $\begin{array}{c}\text { Not } \\
\text { intervening }\end{array}$ \\
\hline 3 & $\begin{array}{l}\text { Independent Commissioner -> } \\
\text { Corporate Value through } \\
\text { Economic Value Added as an } \\
\text { Intervening variable }\end{array}$ & $-0,442$ & $-0,0433$ & Intervening \\
\hline 4 & $\begin{array}{l}\text { Audit Committee-> Corporate } \\
\text { Value through Economic Value } \\
\text { Added as an Intervening variable }\end{array}$ & $-0,168$ & $-0,031$ & Intervening \\
\hline 5 & $\begin{array}{l}\text { Managerial Ownership -> } \\
\text { Corporate Value through } \\
\text { Economic Value Added as an } \\
\text { Intervening variable }\end{array}$ & $-0,083$ & $-0,019$ & Intervening \\
\hline 6 & $\begin{array}{l}\text { Institutional Ownership -> } \\
\text { Corporate Value through } \\
\text { Economic Value Added as an } \\
\text { Intervening variable }\end{array}$ & 0,301 & $-0,0152$ & Not \\
\hline 7 & $\begin{array}{l}\text { SRDI -> Corporate Value } \\
\text { through Economic Value Added } \\
\text { as an Intervening variable }\end{array}$ & 0,242 & 0,0612 & Not \\
intervening
\end{tabular}

Source: processed data, 2017 


\section{CONCLUSION}

Based on calculation above, it can be concluded that:

1. The structure of Good Corporate Governance and Sustainability Report Dislosure simultaneously affects the Economic Value Added.

2. The structure of Good Corporate Governance has an effect on the Economic Value Added.
3. Sustainability Report Disclosure has an effect on Economic Value Added.

4. The structure of Good Corporate Governance simultaneously influences the value of the company.

5. The structure of Good Corporate Governance affects the value of the company.

6. Effect of Economic Value Added on Company Values

\section{REFERENCES}

Al-Mamun, Abdullah. (2014).Relationship Between Audit Committee Characteristics, External Auditors And Economic Value Added (Eva) Of Public Listed Firms In Malaysia. Corporate Ownership \& Control / Volume 12, Issue 1, 2014, Continued - 9

Botosan , Christine A.(2006)..Disclosure and the cost of capital: what do we know?. Accounting and Business Research, Intemational Accounting Policy Forum, pp. 31-40. 2006

Bukhori, Iqbal \& Raharja. (2012). Pengaruh Good Corporate Governance dan Ukuran Perusahaan Terhadap Kinerja Perusahaan (Studi Empiris pada Perusahaan yang Terdaftar di BEI 2010). Diponegoro Journal of Accounting

Debby et al. (2014). Good Corporate Governance, Company's Characteristics and Firm Value: Empirical Study of Listing Banking on Indonesia Stock Exchange. GSTF Journal on Business Review (GBR) 3 (4): 81-88.

Ernest \& Young, Boston College Center for Corporate Citizenship. (2013). Value of Sustainability Reporting.
Fama, Eugene $\mathrm{F}$ and French, Kenneth R.(1998). Taxes, Financing Decision, and Firm Value, The Journal of Finance; Vol. LIII No. 3, June, pp. 819-843.

Fatchan, Ilham Nuryana.(2016). Pengaruh Corporate Governance Pada Hubungan Sustainability Report Dan Nilai Perusahaan Studi Empiris Perusahaan Go Public Di Indonesia Periode 2013-2014. Skripsi. Universitas Muhammadiyah Surakarta.

Ghozali, I. \& Chariri, A. (2007). Teori Akuntansi. Semarang: Badan Penerbit Universitas Diponegoro

Habbash, Murya.(2016).Corporate Governance And Corporate Social Responsibility Disclosure: Evidence From Saudi Arabia. Journal of Economic and Social Development, Vol. 3, No. 1, 2016.

Hadi, Nor. (2011). Corporate Social Responsibility.Yogyakarta:Graha Ilmu.

Handoko, W. (2008). Pengaruh Economic Value Added, ROE, ROA dan EPS Terhadap Perubahaan Harga Saham Perusahaan Kategori LQ-45 pada Bursa Efek Jakarta. Skripsi 
Economics and Accounting Journal

Vol. 1, No. 3, September 2018

Fakultas Ekonomi, Universitas Muhammadiyah Surakarta.

Khairiyani, Sri Rahayu, Netty Herawaty.(2016). Pengaruh Struktur Kepemilikan dan Struktur Pengelolaan Terhadap Kinerja Keuangan Serta Implikasinya Terhadap Nilai Perusahaan pada Perusahaan LQ 45 di Bursa Efek Indonesia Tahun 2012-2014. Simposium Nasional Akuntansi XIX, Lampung,

Murwaningsari., Etty. (2009). Hubungan Corporate Governance, Corporate Social Responsibilities dan Corporate Financial Performance Dalam Satu Continuum. Jurnal Akuntansi Dan Keuangan, Vol. 11, No. 1, Mei 2009: 30-4.

Putri, Ni Made, Sri Rahayu dan I Made Dana. (2016). Pengaruh EVA, MVA Dan Likuiditas Terhadap Harga Saham Pada Perusahaan Food and Beverages. E-Jurnal Manajemen Unud, Vol. 5, No.1, 2016: 443 - 469 .

Safitri, Dian Anggraeni.(2015). Sustainability Report Terhadap Kinerja Keuangan Dan Pasar. Jurnal Ilmu \& Riset Akuntansi Vol. 4 No. 4 (2015).Sekolah Tinggi Ilmu Ekonomi Indonesia (STIESIA) Surabaya.

Soelistyoningrum, J.N. (2011). Pengaruh Pengungkapan Sustainability Report Terhadap Kinerja Keuangan. Skripsi. Universitas Diponegoro. Semarang.

Sugiyono. (2009). Metode Penelitian Bisnis. Bandung: Alfabeta.

Suhartanti, Tutut dan Nur Fadjrih Asyik.(2015). Pengaruh Corporate Governance Terhadap Nilai Perusahaan Dengan Kinerja Keuangan Sebagai Variabel Moderating. Jurnal Ilmu \& Riset Akuntansi Vol. 4 No. 8 (2015).

Tarigan,Josua, Hatane Semuel.(2014).Pengungkapan Sustainability Report dan Kinerja
Keuangan. Jurnal Akuntansi dan Keuangan, Vol. 16, No. 2, November 2014, 88-101.

Utomo, Lisa Linawati.(1999). Economic Value Added Sebagai Ukuran Keberhasilan Kinerja Manajemen Perusahaan. Jurnal Akuntansi dan Keuangan Vol. 1, No. 1, Mei 1999 : $28-42$

Wahyudi, Untung, Hartini Prasetyaning Pawestri..(2006). Implikasi Struktur Kepemilikan Terhadap Nilai Perusahaan: Dengan Keputusan Keuangan Sebagai Variabel Intervening. Simposium Nasional Akuntansi 9 Padang.

Wedayanthi, Komang Krisna, Ni Putu Ayu Darmayanti.(2016). Pengaruh Economic Value Added, Komposisi Dewan Komisaris Independen Dan Return On Assets Terhadap Nilai Perusahaan. E-Jurnal Manajemen Unud, Vol. 5, No. 6, 2016: 36473676.

Wibowo, Santoso, Yokhebed ,Lambok DR Tampubolon.(2016). Pengaruh CSR Disclosure Dan GCG Terhadap Nilai Perusahaan Dengan Kinerja Keuangan Sebagai Variabel Intervening Di Perusahaan Manufaktur Yang Terdaftar Di Bursa Efek Indonesia (2012-2014). Prosiding Seminar Nasional Multi Disiplin Ilmu \& Call For Papers Unisbank (Sendi_U) Ke-2 Tahun 2016.

Yu, Kun, Shuili Du, C.B. Bhattacharya Everybody's Talking But is Anybody Listening? Stock Market Reactions to Corporate Social Responsibility Communications.http://www.hbs.ed u/faculty/conferences/2013sustainab ilit-

andcorporation/Documents/stock_m arket_reactions_to_CSR_reports_A ug31.pdf

www.idx.co.id

www.sahamok.co.id 